\title{
Non-isothermal crystallization and melting of ethylene-vinyl acetate copolymers with different vinyl acetate contents
}

\author{
X. M. Shi, J. Zhang*, J. Jin, S. J. Chen \\ Department of Polymer Science and Engineering, College of Materials Science and Engineering, Nanjing University of \\ Technology, Nanjing 210009, People's Republic of China
}

Received 25 May 2008; accepted in revised form 20 July 2008

\begin{abstract}
Non-isothermal crystallization and subsequent melting of three grades of ethylene-vinyl acetate copolymer were investigated by differential scanning calorimetry (DSC) technique. The DSC crystallization curves show that vinyl acetate (VAc) content has the same effect on the onset, peak and final crystallization temperatures, and these copolymers have almost the same spacing of thermal windows under identical crystallization condition. Subsequent melting DSC results suggest that EVA14 (14 wt\% VAc) has the narrowest distribution of lamellar thickness and the most perfect crystals. Though the instantaneous nucleation was preferred, non-isothermal crystallization kinetics shows that EVA14 could form tridimensional crystallites, whereas EVA18 (18 wt\% VAc) and EVA28 (28 wt\% VAc) are prone to crystallize two-dimensionally, as a result of more noncrystallizable VAc co-monomers introduced in the crystallizable ethylene segments. The growth rate falls on the following sequence: EVA14>EVA18>EVA28. Moreover, the kinetic crystallizability $G$ also well characterizes the variation of the non-isothermal crystallization of these EVA materials, in the view of structural impediment caused by the VAc content.
\end{abstract}

Keywords: thermal properties, ethylene-vinyl acetate copolymer (EVA), crystallization and melting, crystallization kinetics

\section{Introduction}

The crystallization and melting behavior of semicrystalline polymers has been studied for many years, but there is still much work to be done due to the differences in the molecular structure for different polymers. The reaction rate constant does not depend on temperature in case of isothermal measurement, while during non-isothermal experiment the value of rate constant depends on temperature. In these cases the results are less reliable, but the non-isothermal crystallization behavior is of more practical interest than isothermal behavior. This is because polymers are usually processed under nonisothermal cycles rather than under isothermal condition.
Ethylene-vinyl acetate copolymer (EVA) is a random copolymer synthesized from ethylene monomer and vinyl acetate (VAc) co-monomer. The copolymer is often produced by continuous bulk polymerization under high pressure similarly to low density polyethylene (LDPE) [1]. Its properties are easily variable by the adjustment of the comonomer ratio. When the VAc content is low, the copolymer is more akin to polyethylene and has enough ability to crystal, but for high VAc content the pendent acetoxy is excessive enough and prevents adjacent polyethylene chains packing into the crystal lattice [2], then totally amorphous and rubber-like EVA is expected when the VAc content is more than $43 \%$ by weight as indicated by Kamath and Wakefield [3]. It should be noticed that Varga

*Corresponding author, e-mail: zhangjun@ njut.edu.cn

(C) BME-PT and GTE 
and his coworkers revealed that the crystallization and melting characteristics of PE are very sensitive to the VAc content [4].

In the present discussion, the non-isothermal crystallization kinetics for three different grades of EVA was investigated by differential scanning calorimetry (DSC) technique. The main objective for this work is to study the effect of VAc comonomer content on the crystallization behavior of these copolymers. The non-isothermal DSC crystallization and melting curves of these copolymers were considered and the derived kinetic parameters were analyzed based on Avrami equation and its modified formulation. The kinetic crystallizability was also employed to evaluate the crystallization differences arising from the influence of VAc content on the EVA structure.

\section{Experimental}

\subsection{Materials}

Three grades of ethylene-co-vinyl acetate copolymer (EVA) with varying amounts of vinyl acetate (VAc) content are given in Table 1. EVA14 and EVA18 were supplied by Beijing Organic Chemical Plant, China; while EVA28 was supplied by Sumitomo Chemical Company, Japan. Generally, the VAc co-monomer can be considered to be randomly distributed along the copolymer chains.

\subsection{Differential scanning calorimetry measurements}

The non-isothermal crystallization and the following melting experiments of EVA samples with different VAc contents were carried out with a differential scanning calorimeter (DSC) instrument (model Pyris 1, PerkinElmer, USA). Details of samples as received were listed in Table 1. Samples weighing about $10 \mathrm{mg}$ were cut off for the DSC. Argon purge gas with a flux of $20 \mathrm{ml} / \mathrm{min}$ was used to prevent thermal degradation of samples during the scanning. In order to remove the volatile impu- rities and erase the former thermal history, EVA samples were first heated up to $150^{\circ} \mathrm{C}$ at a rapid heating rate of $20^{\circ} \mathrm{C} / \mathrm{min}$ and kept at $150^{\circ} \mathrm{C}$ for 5 minutes. Then they were cooled down from 150 to $0^{\circ} \mathrm{C}$ at the rate of $5^{\circ} \mathrm{C} / \mathrm{min}$ to obtain the nonisothermal crystallization curves. Finally, the remelting was finished by second heating run from 0 to $150^{\circ} \mathrm{C}$ at $10^{\circ} \mathrm{C} / \mathrm{min}$.

The crystallinity $\left(X_{c}\right)$ of the samples was calculated according to Equation (1):

$X_{c}=\frac{\Delta H_{f}}{\Delta H_{f}^{*}} \cdot 100 \%$

where $\Delta H_{f}^{*}$ is the enthalpy of fusion of the perfect polyethylene (PE) crystal and $\Delta H_{f}$ is the enthalpy of fusion of the EVA samples, respectively. The value of $\Delta H_{f}^{*}$ for PE is $277.1 \mathrm{~J} / \mathrm{g}$ [5].

\section{Results and discussion}

\subsection{Non-isothermal crystallization behavior}

Figure 1 shows the non-isothermal crystallization DSC curves of the three grades of EVA. The corresponding values from the curves are collected in Table 2. As expected, DSC curves exhibit only one crystallization peak, and the crystallization peak

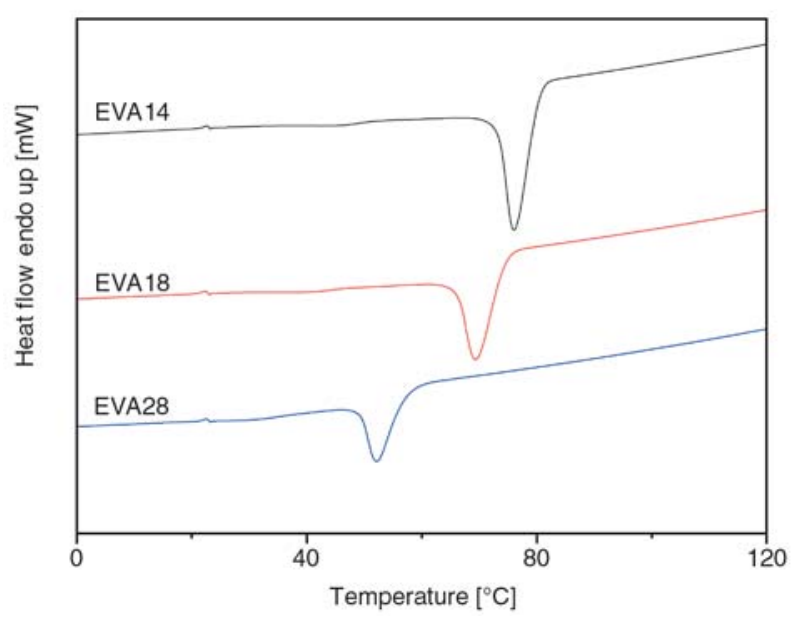

Figure 1. DSC curves of non-isothermal crystallization for the three EVA materials

Table 1. Details of EVA materials used

\begin{tabular}{|c|c|c|c|c|}
\hline Grade & Nominal VAc [wt\%] & Density $\left[\mathrm{g} / \mathrm{cm}^{3}\right]$ & Melt flow rate $[\mathrm{g} / 10 \mathrm{~min}]$ & Nomenclature \\
\hline $14-2$ & 14 & 0.940 & $2^{\mathrm{a}}$ & EVA14 \\
\hline $18-3$ & 18 & 0.935 & $3^{\mathrm{a}}$ & EVA18 \\
\hline KA-31 & 28 & 0.960 & $7^{b}$ & EVA28 \\
\hline
\end{tabular}

aASTM D 1238

bJIS K6924-2 1997 
Table 2. DSC results of non-isothermal crystallization

\begin{tabular}{|c|c|c|c|c|c|}
\hline EVA sample & $\mathbf{T}_{\mathbf{c}}{ }^{\mathbf{o n}}\left[{ }^{\circ} \mathbf{C}\right]$ & $\mathbf{T}_{\mathbf{c}}^{\mathbf{p}}\left[{ }^{\circ} \mathbf{C}\right]$ & $\mathbf{T}_{\mathbf{c}}^{\mathbf{f}}\left[{ }^{\circ} \mathbf{C}\right]$ & $\Delta \mathbf{T}\left[{ }^{\circ} \mathbf{C}\right]$ & $\Delta \mathbf{H}[\mathbf{J} / \mathbf{g}]$ \\
\hline EVA14 & 80.4 & 76.1 & 73.4 & 7.0 & 36.5 \\
\hline EVA18 & 74.6 & 69.4 & 66.2 & 8.4 & 28.7 \\
\hline EVA28 & 57.1 & 52.2 & 49.5 & 7.6 & 17.0 \\
\hline
\end{tabular}

$T_{c}^{o n}$ : onset crystallization temperature; $T_{c}^{p}$ : peak crystallization temperature; $T_{c}^{f}$ : final crystallization temperature; $\Delta T_{c}=T_{c}^{o n}-T_{c}^{f}$; $\Delta H_{c}$ : enthalpy of crystallization

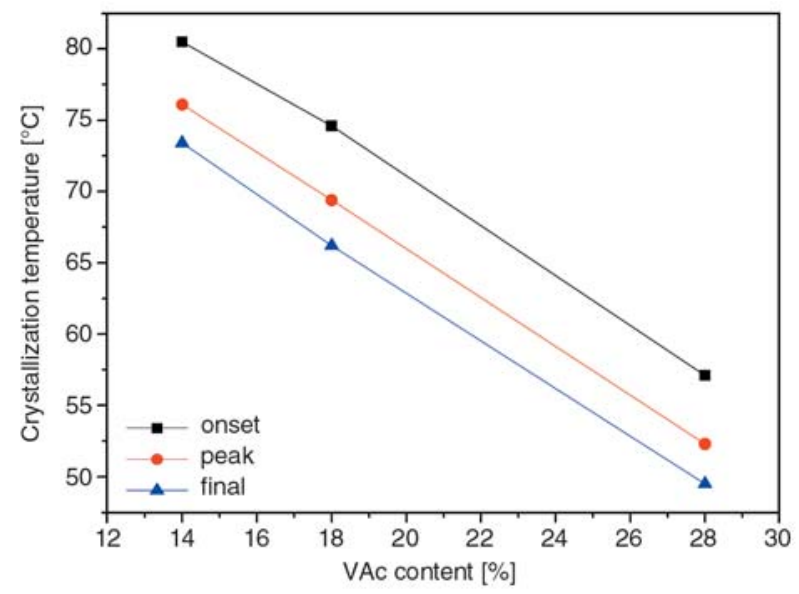

Figure 2. The relationships of crystallization temperatures and VAc content

shifts to lower temperature as the VAc content increases. This suggests that the VAc co-monomer reduces the stereoregularity of polyethylene macromolecular chain and the crystallization is restricted to certain degree. The same tendency of $T_{c}^{o n}, T_{c}^{p}$ and $T_{c}^{f}$ is observed in Figure 2. It is interesting to notice that there are good linear relationships between these crystallization temperatures and the VAc content. The slopes of the onset, peak and final crystallization temperature against VAc content are parallel well with each other, indicating the same effect of VAc content on these crystallization temperatures. However, $\Delta T_{\mathrm{c}}$ difference of these EVA materials is not so large as expected. All these EVA materials have a close $\Delta T_{\mathrm{c}}$ value around $8^{\circ} \mathrm{C}$. It could be inferred that these polymers have the same spacing of thermal windows under the same non-isothermal crystallization condition, independent of difference in VAc contents.

\subsection{Subsequent melting behavior}

To investigate the subsequent melting behavior of these EVA materials after the same non-isothermal crystallization condition, the melting curves were recorded in Figure 3. The characteristic data from the DSC curves are summarized in Table 3. Like the crystallization temperatures above, melting peak and final temperatures decrease accordingly with the increase in VAc content, indicating the lamellar thickness decrease since the melting temperature of a given sample decreases with decrease of lamellar thickness [6]. There is almost $20^{\circ} \mathrm{C}$ in peak melting temperature difference between EVA14 and EVA28. It is also apparent from the melting curves that the melting peak of EVA14 which has the lowest VAc content is the sharpest among the three EVA materials. It has been considered that the width of melting peak can reflect the distribution of the lamellar thickness [7]. Therefore, EVA14 has a narrower distribution of lamellar

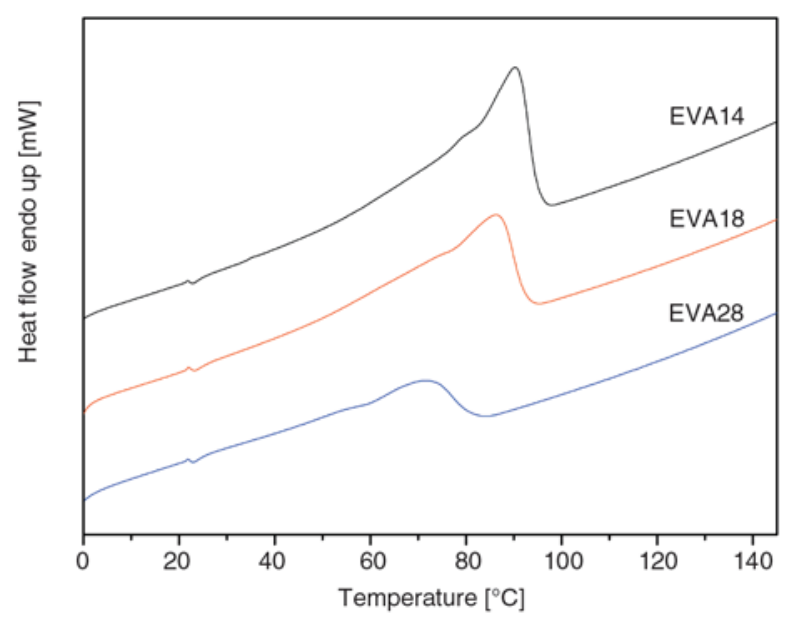

Figure 3. DSC curves of subsequent melting for the three EVA materials

Table 3. DSC results of subsequent melting

\begin{tabular}{|c|c|c|c|c|}
\hline EVA sample & $\mathbf{T}_{\mathbf{m}}^{\mathbf{p}}\left[{ }^{\circ} \mathbf{C}\right]$ & $\mathbf{T}_{\mathbf{m}}^{\mathbf{f}}\left[{ }^{\circ} \mathbf{C}\right]$ & $\Delta \mathbf{H}_{\mathbf{f}}[\mathbf{J} / \mathbf{g}]$ & $\mathbf{X}_{\mathbf{c}}[\boldsymbol{\%}]$ \\
\hline EVA14 & 90.2 & 95.2 & 63.9 & 23.1 \\
\hline EVA18 & 86.0 & 92.5 & 50.6 & 18.3 \\
\hline EVA28 & 70.5 & 81.6 & 21.8 & 7.9 \\
\hline
\end{tabular}

$T_{m}^{p}$ : peak melting temperature; $T_{m}^{f}$ : final melting temperature; $\Delta H_{f}$ : enthalpy of melting; $X_{c}$ : crystallinity 
thickness than that of EVA18 and EVA28. Similarly, the crystallinity confirms the difference of the crystal perfection of these copolymers. The crystallinity of EVA28 is only $7.9 \%$ which is no more than $45 \%$ of EVA18 or no more than $35 \%$ of EVA14. It is worth noting that the onset melting temperatures of these copolymers are difficult to discern because of broad melting range, indicating all of these EVA materials have a wide size distribution of the crystallites. All the results above can be explained based on the amount of total non-crystallizable, intra-molecular defects [7] (i.e. VAc defects). The EVA material with high VAc content should have high amount of the VAc defects, and tend to crystallize imperfectly.

\subsection{Non-isothermal crystallization kinetics}

Crystallization kinetics of a material can be analyzed by evaluating the extent or degree of phase transformation which is often denoted as $X_{t}$. The relative crystallinity $X_{t}$ as a function of time or temperature by recording the heat flow during crystallization process and is defined as Equation (2) $[8,9]$ :

$$
X_{t}=\frac{\int_{T_{0}}^{T} H(T) \mathrm{d} T}{\int_{T_{0}}^{T} H(T) \mathrm{d} T} \cdot 100 \%
$$

where $T_{0}$ and $T_{\infty}$ are the initial and end crystallization temperature, respectively, and $H(T)$ is the heat flow at temperature T. $X_{t}$ is determined from the crystallization curve by integral method.

The relative crystallinity is plotted versus different crystallization temperatures and time as is shown in Figures 4 and 5. At the same cooling rate of $5^{\circ} \mathrm{C} / \mathrm{min}$, development curves for the relative crystallinity of these EVA materials display the same reversed $S$ type as the crystallization temperature goes down, whereas the curves display the similar $S$ type as the crystallization time elapses. Though the three grades of EVA possess different VAc content, the whole crystallization process of these polymers is a similar packing work of the crystallizable ethylene segments between uncrystallizable vinyl acetate segments. So these EVA materials undoubt-

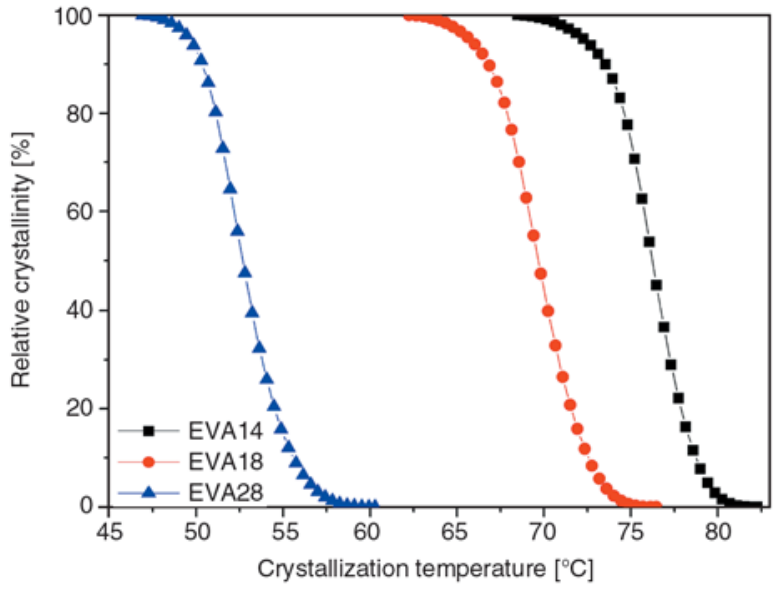

Figure 4. Plots of relative crystallinity vs. crystallization temperature for the three EVA materials

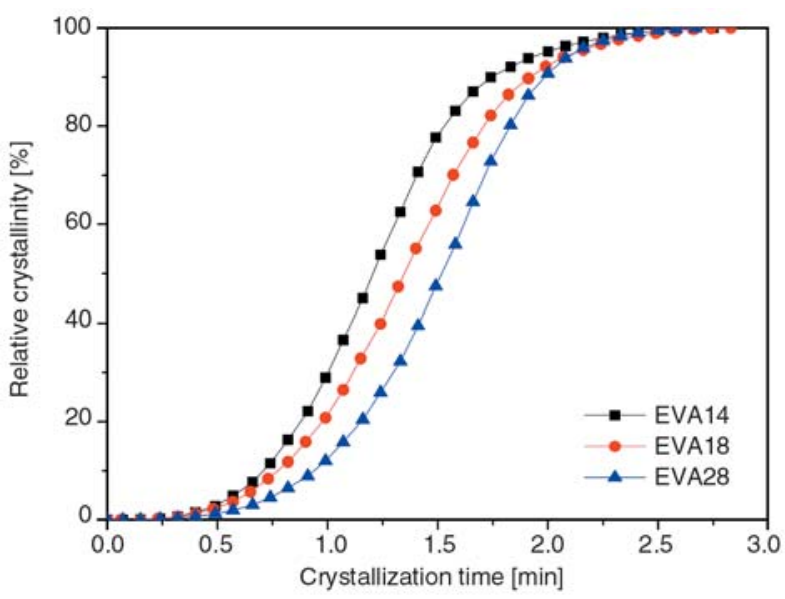

Figure 5. Plots of relative crystallinity vs. crystallization time for the three EVA materials

edly display similar development curves with crystallization temperature or time.

Majority of the proposed formulations for predicting non-isothermal crystallization kinetics are based on the modification of Avrami equation. Avrami equation for non-isothermal crystallization is expressed by Equation (3) [10-12]:

$1-X_{t}=\exp \left(-Z t^{n}\right)$

where the exponent $n$ is a mechanism constant which value depends on the type of nucleation and the growth dimension, and the parameter $Z$ is a growth rate constant involving both nucleation and growth rate parameters [13].

The kinetics parameters of non-isothermal crystallization were determined, based on the assumptions that non-isothermal crystallization may be treated as a sequence of isothermal crystallization steps 


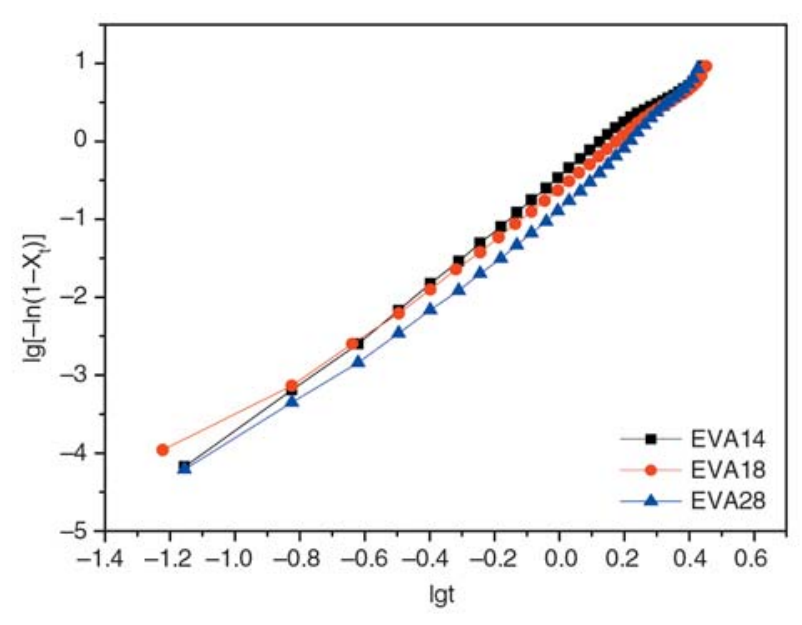

Figure 6. Plots of $\lg \left[-\ln \left(1-X_{t}\right)\right]$ vs. $\lg t$ for the three EVA materials

$[14,15]$. In this case, Equation (3) can be transformed into Equation (4):

$$
\lg \left[-\ln \left(1-X_{t}\right)\right]=\lg Z+n \lg t
$$

Figure 6 gives the plots of $\lg \left[-\ln \left(1-X_{t}\right)\right]$ versus $\lg t$, they show relatively good linear relationship (the correlation coefficient for linear regression, $R>0.98$ for all these curves). However, all the three straight lines terminate with an upward tail, which is considered as the secondary crystallization as observed in other polymers [16-18]. The Avrami exponent $n$ and constant $Z$ can be obtained from the slope and the intercept of the plot of $\lg \left[-\ln \left(1-X_{t}\right)\right]$ against $\lg t$. Considering the non-isothermal character of the process investigated, the growth rate constant is corrected by the cooling rate $\phi$, then the final form of growth rate constant $Z_{c}$ is obtained from Equation (5) [14]:

$$
\lg Z_{c}=\frac{\lg Z}{\phi}
$$

where $n$ and $Z_{c}$ values are listed in Table 4 . It is found that the Avrami exponent of EVA14 is between 3 and 4, whereas the Avrami exponents of EVA18 and EVA28 are around 3. It seems that there may be differences in the nucleation and growth mechanisms of these EVA materials.
EVA14 could form coexistent tridimensional crystallites from instantaneous and sporadic nucleation $(n=3.33)$. In the case of EVA18 and EVA28, the Avrami exponents imply two possibilities: the two EVA materials may form tridimensional crystallites from sporadic nucleation; or they probably form two-dimensionally growing crystallites from instantaneous nucleation $[19,20]$. Since no other heterogeneous material was introduced, the instantaneous nucleation was preferred for the crystallization of the three EVA materials. Consequently, EVA14 is prone to form tridimensional crystallites as polyethylene material often does, whereas EVA18 and EVA28 are forced to crystallize in twodimensional caused by the impediment of more acetoxy side chains. In addition, the lowest half crystallization time $\left(t_{0.5}\right)$ of EVA14 indicate the relative fast crystallization compared with EVA18 and EVA28. The same conclusion can also be drawn from the comparison of the growth rate constant $Z_{c}$.

To characterize the kinetics of non-isothermal crystallization of PET, Jeziorny [14] used a parameter $G$, known as 'the kinetic crystallizability'. It characterizes the degree of transformation obtained over the entire crystallization range $\left(T_{m}, T_{g}\right)$ with unit cooling rate. The calculation of the parameter is done by Equation (6):

$$
G=\left(\frac{\pi}{\ln 2}\right)^{\frac{1}{2}} \frac{\int_{t_{0}}^{t_{\max }} H_{t} \mathrm{~d} t}{\int_{t_{\max }}^{t_{f}} H_{t} \mathrm{~d} t} \frac{1}{t_{\max }} \frac{D}{2}
$$

where $t_{0}, t_{\max }, t_{f}$ are the time corresponding to $T_{c}^{o n}$, $T_{c}^{p}$ and $T_{c}^{f}$, respectively; $D$ is the half width of the crystallization curve.

This parameter, like the growth rate constant $Z$ above, requires proper correction. The final form of this parameter is shown in Equation (7):

$$
G_{c}=\frac{G}{\phi}
$$

Table 4. Parameters for the non-isothermal crystallization of the three EVA materials

\begin{tabular}{|c|c|c|c|c|}
\hline EVA sample & $\mathbf{n}$ & $\mathbf{t}_{\mathbf{0 . 5}}[\mathbf{m i n}]$ & $\mathbf{Z}_{\mathbf{c}}\left[\mathbf{m i n}^{-\mathbf{n}}\right]$ & 0.80 \\
\hline EVA14 & 3.33 & 1.20 & $\mathbf{G}_{\mathbf{c}}$ \\
\hline EVA18 & 2.96 & 1.35 & 0.77 & 1.697 \\
\hline EVA28 & 2.96 & 1.53 & 0.72 & 1.659 \\
\hline
\end{tabular}


$G_{c}$ values of these EVA materials are listed in Table 4. It is quite evident that the $G_{c}$ value decreases with an increase in VAc content. Jeziorny [14] recommended $G_{c}$ for characterizing the kinetics of non-isothermal crystallization, because this parameter aims at the determination of quantitative relations between the kinetics of polymer crystallization and the structural features. From the data in Table 4, it is found that the $G_{c}$ value of EVA18 is very close to that of EVA14, and the $G_{c}$ value of EVA28 is relatively low due to the high VAc content compared with the other two grades of EVA. It has been reported that the increase in the amount of total co-monomer in EAA or E-MA-AA will enhance the energy barrier for crystallizable segments to crystallize [7]. Since the increase in the amount of the randomly distributed VAc comonomer will result in a decrease in both the number and length of the crystallizable ethylene segment, the EVA28 material which has the most VAc content should have the biggest energy barrier for the ethylene segments to overcome. This is also consistent with the fact that bulky nature of the acetoxy side chain will reduce the ability of the polymer chains to pack closely together to form crystallites [1]. It can be thought from the results above that the VAc content undoubtedly has a negative effect on the crystal growth of EVA material as a result of the strong dependence of the copolymer structure on the VAc co-monomer.

\section{Conclusions}

The non-isothermal crystallization for the three grades of EVA materials indicates that the crystallization tendency of EVA decreases with increasing of VAc content. VAc content has the same effect on the onset, peak and final crystallization temperatures. And with increasing of VAc content, perfection of EVA crystals decreases.

In addition, the results of non-thermal crystallization kinetics show that the simplified assumption of a sequence of isothermal crystallization is suitable for characterizing the non-isothermal crystallization of these EVA materials. Though the instantaneous nucleation was preferred, there is difference for the growth of these EVA materials. EVA14 could form tridimensional crystallites whereas EVA18 and EVA28 are prone to crystallize in twodimensional as a result of more noncrystallizable
VAc co-monomers introduced in the crystallizable ethylene segments. The growth rate falls on the following sequence: EVA14>EVA18>EVA28. The kinetic crystallizability $G$ also well characterizes the difference of the non-isothermal crystallization of these EVA materials, in the view of structural impediment caused by the VAc content.

\section{References}

[1] Whelan A., Lee K. S.: Developments in rubber technology, 3. Thermoplastic rubbers. Applied Science Publishers, London (1982).

[2] Henderson A. M.: Ethylene-vinyl acetate (EVA) copolymers: A general review. IEEE Electrical Insulation Magazine, 9, 30-38 (1993).

[3] Kamath P. M., Wakefield R. W.: Crystallinity of ethylene-vinyl acetate copolymers. Journal of Applied Polymer Science, 9, 3153-3160 (1965).

[4] Varga J., Menczel J., Solti A.: The melting of highpressure polyethylene subjected to stepwise heat treatment. Journal of Thermal Analysis and Calorimetry, 17, 333-342 (1979).

[5] Brandrup J., Immergut E. H., Grulke E. A.: Polymer handbook. Wiley-Interscience, New York (1999).

[6] Wunderlich B.: Macromolecular physics. Academic Press, New York (1976).

[7] Somrang N., Nithitanakul M., Grady B. P., Supaphol P.: Non-isothermal melt crystallization kinetics for ethylene-acrylic acid copolymers and ethylene-methyl acrylate-acrylic acid terpolymers. European Polymer Journal, 40, 829-838 (2004).

[8] Bhattarai N., Kim H. Y., Cha D. I., Lee D. R., Yoo D. I.: Nonisothermal crystallization and melting behavior of the copolymer derived from $p$-dioxanone and poly(ethylene glycol). European Polymer Journal, 39, 1365-1375 (2003).

[9] Mya K. Y., Pramoda K. P., He C. B.: Crystallization behavior of star-shaped poly(ethylene oxide) with cubic silsesquioxane (CSSQ) core. Polymer, 47, 5035-5043 (2006).

[10] Avrami M.: Kinetics of phase change I. General theory. Journal of Chemical Physics, 7, 1103-1112 (1939).

[11] Avrami M.: Kinetics of phase change, II. Transformation-time relations for random distribution of nuclei. Journal of Chemical Physics, 8, 212-224 (1940).

[12] Xiong H., Gao Y., Li H. M.: Non-isothermal crystallization kinetics of syndiotactic polystyrene-polystyrene functionalized SWNTs nanocomposites. Express Polymer Letters, 1, 416-426 (2007).

[13] Kong X., Yang X., Zhou E., Ma D.: Nonisothermal crystallization kinetics of ethylene terephthalate-ethylene oxide segmented copolymers with two crystallizing segments. European Polymer Journal, 36, 10851090 (2000) 
[14] Jeziorny A.: Parameters characterizing the kinetics of the non-isothermal crystallization of poly(ethylene terephthalate) determined by DSC. Polymer, 19, 1142-1144 (1978).

[15] Kalam M. R., Chu E.: Isothermal and nonisothermal crystallization of polyethylene. Polymer Engineering and Science, 23, 27-31 (1983).

[16] Liu T. X., Mo Z., Wang S. G., Zhang H.: Nonisothermal melt and cold crystallization kinetics of poly(aryl ether ether ketone ketone). Polymer Engineering and Science, 37, 568-575 (1997).

[17] Run M. T., Yao C. G., Wang Y. J.: Morphology, isothermal and non-isothermal crystallization kinetics of poly(methylene terephthalate). European Polymer Journal, 42, 655-662 (2006).
[18] Maa Y-L., Hu G-S., Ren X-L., Wang B-B.: Nonisothermal crystallization kinetics and melting behaviors of nylon 11/tetrapod-shaped $\mathrm{ZnO}$ whisker (T-ZnOw) composites. Materials Science and Engineering, Part A: Structural Materials Properties Microstructure and Processing, 460-461, 611-618 (2007).

[19] Gupta A. K., Rana S. K., Deopura B. L.: Crystallization kinetics of high-density polyethylene/linear lowdensity polyethylene blend. Journal of Applied Polymer Science, 51, 231-239 (1994).

[20] Xu X., Xu J., Chen L., Liu R., Feng L.: Nonisothermal crystallization kinetics of ethylene-butene copolymer/ low-density polyethylene blends. Journal of Applied Polymer Science, 80, 123-129 (2001). 